\title{
Identification of HIV patients with active pulmonary tuberculosis using urine based polymerase chain reaction assay
}

Antonio Aceti, Stefania Zanetti, Maria S Mura, Leonardo A Sechi, Franco Turrini, Franca Saba, Sergio Babudieri, Franca Mannu, Giovanni Fadda
Institute of Infectious Diseases, University of Sassari, 07100 Sassari, Italy

A Aceti

M S Mura

F Saba

S Babudieri

Department of Biomedical Sciences, Section of

Experimental and

Clinical Microbiology,

University of Sassari,

Sassari, Italy

S Zanetti

L A Sechi

Department of Genetics, Biology and Medical Chemistry, University of Turin, Turin, Italy

F Turrini

F Mannu

Institute of

Microbiology, Faculty of Medicine, Catholic University, Rome, Italy G Fadda

Correspondence to: Professor A Aceti.

Received 29 May 1998 Returned to author 12 August 1998 Revised manuscript received 10 November 1998 Accepted for publication 11 November 1998

\begin{abstract}
Background-Despite the increased dissemination of tuberculosis among HIV infected patients, the diagnosis is difficult to establish. Traditional microbiological methods lack satisfactory sensitivity. We have developed a highly sensitive and specific nested polymerase chain reaction (PCR) capable of detecting Mycobacterium tuberculosis DNA in urine specimens and have used this test to examine urine specimens from HIV patients with active pulmonary tuberculosis.

Methods-Urine specimens from 13 HIV infected patients with microbiologically proven active pulmonary tuberculosis, 10 AIDS patients with non-tuberculous mycobacterial infection (documented by blood culture), 53 AIDS patients with no evidence of mycobacterial disease, and $\mathbf{8 0}$ healthy subjects ( 25 with positive skin test to purified protein derivative) were tested for $M$ tuberculosis using PCR, acid fast staining (AFS), and culture.

Results-Of the urine specimens from patients with active tuberculosis, all tested positive by PCR, two by culture, and none by AFS. No reactivity was observed in urine specimens from patients with nontuberculous mycobacterial infection. Of the 53 AIDS patients without mycobacterial infection, one had a positive urine PCR. Normal subjects were all negative.

Conclusions-Urine based nested PCR for $M$ tuberculosis may be a useful test for identifying HIV patients with pulmonary tuberculosis.

(Thorax 1999;54:145-146)
\end{abstract}

Keywords: tuberculosis; urine; polymerase chain reaction; AIDS

Tuberculosis is the most common serious HIV related infection worldwide, but decreased reactivity to purified protein derivative (PPD), atypical presentations, and confusion with other HIV related infections hamper the diagnosis of tuberculosis in AIDS patients. ${ }^{1}$ Traditional microbiological methods are complex and time consuming and they lack satisfactory sensitivity. Genomic detection by polymerase chain reaction (PCR) has been reported to be a rapid, simple, and very sensitive tool for detecting mycobacteria in clinical samples. ${ }^{2}$ We have developed a highly sensitive and specific nested PCR capable of detecting $M$ tuberculosis DNA in urine specimens. ${ }^{4}$ We have recently examined urine specimens from HIV positive patients with active pulmonary tuberculosis using this technique.

\section{Methods}

Thirteen HIV infected patients (nine men) of mean (SD) age 28 (5) years (range 24-35) admitted to the Institute of Infectious Diseases of the University of Sassari, Italy with microbiologically proven pulmonary tuberculosis (positive sputum by direct examination and/or culture) were studied. The median CD4 count was $165 \times 10^{6} / 1$ (range 100-306). None had clinical or biochemical evidence of genitourinary disease. Ten patients with AIDS with a non-tuberculous mycobacterial infection documented by blood culture (median CD 4 count $45 \times 10^{6} / 1$ range $5-87$ ) were also studied, and 53 consecutive AIDS patients with no evidence of mycobacterial disease on the chest radiograph and on blood, stool and urine cultures (median CD4 count $97 \times 10^{6} / 1$; range 18-195). None of these patients had previously been treated for tuberculosis. Eighty healthy subjects (25 with positive skin test to PPD) served as controls.

After informed consent was obtained, morning urine was collected from patients and controls for three consecutive days. The three urine specimens taken from each subject were combined and centrifuged at $3000 \mathrm{~g}$ for 20 minutes, the supernatant was removed, and the pellet was resuspended in $2 \mathrm{ml}$ of $\mathrm{NaOH} 4 \%$. After incubation for 15 minutes $40 \mathrm{ml}$ of phosphate buffered saline (PBS) were added and the samples again centrifuged at $3000 \mathrm{~g}$ for 20 minutes. The pellets were retained, resuspended in $1 \mathrm{ml}$ of PBS, and aliquots of $100 \mu \mathrm{l}$ were stored at $-80^{\circ} \mathrm{C}$ for analysis by PCR. Urine based PCR was carried out according to the method previously described. ${ }^{4}$ Two sets of primers specific for IS6110 were selected; outer primers were TB290 (5'-GGCGGGACAA- 
Table 1 Positive results for $M$ tuberculosis obtained by testing urine specimens from different groups of subjects using PCR, culture method, and acid fast staining (AFS)

\begin{tabular}{lllll}
\hline Group & No. & PCR positive & Culture positive & AFS positive \\
\hline HIV patients with tuberculosis & 13 & $13(100 \%)$ & $2(15.4 \%)$ & 0 \\
HIV patients with non-tuberculous & 10 & 0 & 0 & 0 \\
$\quad$ mycobacteriosis & 53 & $1(1.8 \%)$ & 0 & 0 \\
HIV patients with no mycobacteriosis & 80 & 0 & 0 & 0 \\
Healthy subjects & &
\end{tabular}

CGCCGAATTGCGAA) andTB856 (5'-CGAGCGTAGGCGTCGGTGACAAAG); inner primers were TB431 (5'-TACTACGACCACATCAACCG) and TB740 (5'-CGCTGATCCGGCCACAGCCC). The sensitivity of nested PCR, estimated by diluting the H37Rv chromosomal DNA, was 1 equivalent genome. No amplification product was obtained with $M$ avium, $M$ fortuitum, $M$ xenopii, $M$ kansasii, $M$ malmoense, or $M$ chelonei. The identity of the PCR products was demonstrated by performing different Southern blots using the IS6110 as a probe against the amplified products from various species of mycobacteria.

Urine specimens were tested for $M$ tuberculosis by direct examination using acid fast staining (AFS) and by culture on the Bactec system (Bactec 460 TB, Beckton Dickinson).

The study was approved by the ethics committee of the Faculty of Medicine of the University of Sassari.

\section{Results}

Of 13 HIV patients with pulmonary tuberculosis, all had urine positive for $M$ tuberculosis by nested PCR, only two (15.4\%) by the culture method, and none by AFS. No positivity was observed in urine specimens from patients with non-tuberculous mycobacterial infection. Of 53 AIDS patients without clinical evidence of mycobacterial infection, one $(1.8 \%)$ had a positive urine PCR. Normal subjects were all negative (table 1 ).

\section{Discussion}

The results of this study establish the usefulness of the PCR assay for the rapid detection of $M$ tuberculosis in urine samples from HIV positive patients. Indeed, by using this technique we could detect mycobacterial DNA in all the cases of pulmonary tuberculosis we studied and no false positive results were seen in patients infected with mycobacteria other than $M$ tuberculosis. The AIDS patient with a positive urine PCR without clinical and microbiological evidence of tuberculosis at the time of the study developed a disseminated tuberculosis two months later. It is possible that this technique, with its very high level of sensitivity, can identify the infection before it is clinically diagnosed. It has been reported that a nested PCR based on the IS6110 was able to amplify
DNA from mycobacteria other than $M$ tuberculosis. ${ }^{6}$ However, none of the other mycobacteria tested in our study gave amplification products when assayed with PCR. This may be because of the higher specificity of the primers we selected.

Previous studies have shown that, except in patients with genitourinary disease, submission of urine specimens to the traditional microbiology laboratory for identification of $M$ tuberculosis is rarely useful in the diagnosis of tuberculosis. $^{78}$

$M$ tuberculosis has recently been detected in the blood from 39 of 41 patients with proven pulmonary tuberculosis using DNA amplification. ${ }^{9}$ This finding provides evidence that mycobacteria can enter the circulation even in the absence of clinical signs of dissemination. It is therefore conceivable that these bacteria may be cleared from the blood stream in the urine. Indeed, in our study all the patients with pulmonary tuberculosis tested positive by urine PCR analysis. It therefore appears that the organism may be present in the urine of all patients with active tuberculosis, or at least in those with AIDS. Because of the clearance of non-viable organisms and/or the very low bacterial load in urine specimens, mycobacteria cannot be detected using conventional methods. The development of technologies such as DNA amplification would have a significant effect on the diagnosis of tuberculosis. Urine samples are easily collected and any mycobacteria present can be concentrated by centrifugation and promptly and effectively detected by DNA amplification.

This work was supported in part by grants from the project
"Tubercolosi" of the Istituto Superiore di Sanità, Rome, and by grants from Assessorato alla Sanità, Regione Sardegna, Italy.

1 Dolin PJ, Raviglione MC, Kochi A. Global tuberculosis incidence and mortality during 1990-2000. Bull World Health Org 1994;72:213-30.

2 Wilson SM, McNerney R, Nye P, et al. Progress toward a implified polymerase chain reaction and its application to diagnosis of tuberculosis. F Clin Microbiol 1993;30:776-82.

3 Shah S, Miller A, Mastellone A, et al. Rapid diagnosis of tuberculosis in various biopsy and body fluid specimens by the AMPLICOR Mycobacterium tuberculosis polymerase chain reaction. Chest 1998;113:1190-4.

4 Sechi LA, Pinna MP, Sanna A, et al. Detection of Mycobacterium tuberculosis. by PCR analysis of urine and other clinical samples from AIDS and non-HIV-infected patients. Mol Cell Probes 1997;11:281-5.

5 Telenti A, Marchesi F, Balz M, et al. Rapid identification to the species level by polymerase chain reaction and restricthe species level by polymerase chain reaction and res
tion enzyme analysis. $\mathcal{F}$ Clin Microbiol $1993 ; 31: 175-8$.

6 Kent L, McHugh TD, Billington $\mathrm{O}$, et al. Demonstration of homology between IS 6110 of Mycobacterium tuberculosis and DNAs of other Mycobacterium spp. $\mathcal{F}$ Clin Microbiol 1995;33:2290-3

7 Bentz RR, Dimcheff DG, Nemiroff MJ, et al. The incidence of urine cultures positive for Mycobacterium tuberculosis in a general tuberculosis patient population. Am Rev Respir Dis 1975;111:647-50.

8 Mortier E, Pouchot J, Girard L, et al. Assessment of urine analysis for the diagnosis of tuberculosis. BMF 1996;312: 27-8.

9 Condos R, McClune A, Rom WN, et al. Peripheral blood based PCR assay to identify patients with active pulmonary tuberculosis. Lancet 1996;347:1082-5. 